Check for updates

Cite this: RSC Adv., 2019, 9, 31812

\title{
An efficient Au catalyst supported on hollow carbon spheres for acetylene hydrochlorination
}

\author{
Lihua Kang ${ }^{\mathrm{ab}}$ and Mingyuan Zhu (D) *ab \\ Mesoporous hollow carbon spheres (HCSs) were prepared using $\mathrm{SiO}_{2}$ spheres as a hard template, and $\mathrm{Au}$ \\ nanoparticles were then synthesized using $\mathrm{NaBH}_{4}$ as a reducing agent on the surface of the HCS \\ support. Transmission electron microscopy characterization indicated that Au nanoparticles were much \\ smaller on the HCS support than those on the active carbon (AC) support. HCl-TPD showed that the Au/ \\ HCS catalyst displayed a more active site than on Au/AC. The resulting Au/HCS catalyst showed \\ excellent catalytic activity and stability for acetylene hydrochlorination. Acetylene conversion of Au/HCS \\ can be maintained above $92 \%$ even after $500 \mathrm{~h}$ of lifetime. The excellent catalytic performance of Au/ \\ HCS was attributed to the presence of the HCS support, which limited the aggregation of Au nanoparticles.
}

Received 2nd September 2019 Accepted 29th September 2019

DOI: 10.1039/c9ra06989e

rsc.li/rsc-advances polyaniline exhibited remarkable stability for acetylene hydrochlorination. ${ }^{6}$ In our previous work, we reported that metallic $\mathrm{Au}$ nanoparticles exhibited considerable catalytic activity for acetylene hydrochlorination, and the aggregation of Au nanoparticles was another reason for the deactivation of the Aubased catalyst in addition to the reduction of $\mathrm{Au}$ (III) or $\mathrm{Au}(\mathrm{I}){ }^{7}$ Therefore, this study used metallic $\mathrm{Au}$ as an active site for acetylene hydrochlorination. The use of nitrogen-doped carbon may be a good strategy for supports to inhibit the aggregation of Au nanoparticles and promote support stability for acetylene hydrochlorination. ${ }^{8,9}$ Recently, Yuan et al. reported that metallic $\mathrm{Au}(0)$ was directly involved in the catalysis of acetylene hydrochlorination due to the strong mediating properties of $\mathrm{Ce}(\mathrm{Iv}) /$ $\mathrm{Ce}(\mathrm{III})$ with one-electron complementary redox coupling reactions. Thus, the ceria promotion to Au catalysts gives enhanced activity and stability. ${ }^{10}$ The reduction of oxidative Au cations and aggregation of Au nanoparticles cannot be avoided under the acetylene hydrochlorination reaction conditions-especially in long-term testing. Thus, another good strategy to improve catalyst stability may be the use of an appropriate carbon support for $\mathrm{Au}$ catalysts. We found that larger AC pore sizes increased the speed of the reaction and efficiently prohibited carbon deposition. ${ }^{11}$ Li et al. ${ }^{12}$ proposed that some of the oxygenated groups on the surface of AC could improve the catalytic activity and stability of Au catalysts.

HCS has been well reported as catalyst supports due to their high surface area, large pore size, and abundant oxygenated groups; ${ }^{13}$ the hollow structure functions act as a barrier to prevent encapsulated $\mathrm{Au}$ nano-particles from aggregation or leaching. ${ }^{14}$ Therefore, we inferred that $\mathrm{Au}$ aggregation could also be restricted during acetylene hydrochlorination, and an Au catalyst with excellent stability could be obtained by depositing Au nanoparticles on HCS support.
${ }^{a}$ College of Chemistry and Chemical Engineering of Yantai University, Yantai, Shandong, 264005, PR China. E-mail: zhuminyuan@shzu.edu.cn; Tel: +86-993-2057277

${ }^{b}$ School of Chemistry and Chemical Engineering of Shihezi University, Shihezi, Xinjiang, 832000, PR China 


\section{Materials and methods}

\subsection{Materials}

Tetraethyl orthosilicate (TEOS), cetyltrimethylammonium bromide (CTAB), resorcinol, formalin, activated carbon (coconut carbon, 40-60 mesh, marked as $\mathrm{AC}$ ), $\mathrm{HAuCl}_{4} \cdot 4 \mathrm{H}_{2} \mathrm{O}$ (with $47.8 \%$ Au content), $\mathrm{NaBH}_{4}, \mathrm{C}_{2} \mathrm{H}_{2}$ (gas, 99\%), and $\mathrm{HCl}$ (gas, 99\%) were used here.

\subsection{Catalyst preparation}

2.2.1 Preparation of the $\mathrm{SiO}_{2}$ template. $\mathrm{SiO}_{2}$ sphere synthesis was performed using a modified Stöber reaction. ${ }^{15}$ Water $(25 \mathrm{~mL}), 63 \mathrm{~mL}$ of 2-propanol, and $13 \mathrm{~mL}$ of ammonia (27\%, aqueous solution) were mixed and heated to $35{ }^{\circ} \mathrm{C}$ in a water bath. Next, $0.6 \mathrm{~mL}$ of TEOS was added dropwise under vigorous stirring for $30 \mathrm{~min}$ to generate the silica seeds. TEOS ( 5 $\mathrm{mL}$ ) was then added dropwise. The reaction mixture was held constant at $35{ }^{\circ} \mathrm{C}$ for $4 \mathrm{~h}$. The $\mathrm{SiO}_{2}$ spheres were separated by centrifugation, washed with ethanol four times, then air dried.

2.2.2 Preparation of mesoporous hollow carbon spheres. Next, $0.8 \mathrm{~g}$ of as-obtained $\mathrm{SiO}_{2}$ spheres was uniformly dispersed in $70 \mathrm{~mL}$ of deionized water by ultrasonication followed by $2.3 \mathrm{~g}$ of CTAB, $0.35 \mathrm{~g}$ of resorcinol, $28 \mathrm{~mL}$ of ethanol, and $0.1 \mathrm{~mL}$ of ammonia. The mixture was heated to $35{ }^{\circ} \mathrm{C}$ in a water bath and stirred for $30 \mathrm{~min}$ to form a homogeneous dispersion. Formalin was then added to the mixture under stirring. The mixture was cooled to room temperature after $6 \mathrm{~h}$ and aged at room temperature overnight without stirring. The resulting silica@resorcinol-formaldehyde ( $\left.\mathrm{SiO}_{2} @ \mathrm{RF}\right)$ was collected by centrifugation, washed with water and ethanol several times, and dried. The $\mathrm{SiO}_{2}$ @RF product was then heated under a nitrogen atmosphere at $5{ }^{\circ} \mathrm{C} \mathrm{min}^{-1}$ from room temperature to $150{ }^{\circ} \mathrm{C}$ and kept at this temperature for $1 \mathrm{~h}$. The temperature was then increased by $5{ }^{\circ} \mathrm{C} \min ^{-1}$ to $800{ }^{\circ} \mathrm{C}$ and held for $2 \mathrm{~h}$. The $\mathrm{SiO}_{2} @ \mathrm{C}$ product was then treated with a $10 \% \mathrm{HF}$ solution to remove the silica and generate the hollow carbon nanoparticles.

2.2.3 The preparation of Au/HCS catalyst. The Au/HCS catalyst was prepared using $\mathrm{NaBH}_{4}$ reduction methods. ${ }^{8}$ About $1.0 \mathrm{~g}$ of the HCS was dispersed in $100 \mathrm{~mL}$ water under sonication for $30 \mathrm{~min}$. A solution of $\mathrm{HAuCl}_{4} \cdot 4 \mathrm{H}_{2} \mathrm{O}(2.1 \mathrm{~mL}, 1 \mathrm{~g} / 100$ $\mathrm{mL}$ ) was added dropwise to the HCS slurry, and then $\mathrm{NaBH}_{4}$ (21 $\mathrm{mL}, 0.1 \mathrm{M}$ ) was added to this suspension under sonication and stirred for $30 \mathrm{~min}$. The resulting mixture was vigorous stirred for $24 \mathrm{~h}$ at room temperature. Finally, the solution was filtered, washed several times with distilled water, dried in a vacuum at $60{ }^{\circ} \mathrm{C}$, and named as the Au/HCS catalyst. For comparison, an $\mathrm{Au} / \mathrm{AC}$ catalyst was prepared with active carbon as support for comparison. The theoretical loadings of Au metal were $1 \%$ in those two catalysts.

\subsection{Catalyst characterization}

Surface area analysis was carried out by $\mathrm{N}_{2}$ adsorptiondesorption at $77 \mathrm{~K}$ on a Micromeritics ASAP 2020 instrument. Samples were degassed at $423 \mathrm{~K}$ for $15 \mathrm{~h}$ before analysis. The Xray diffraction (XRD) data were collected using a Bruker D8 advanced X-ray diffractometer with $\mathrm{Cu}-\mathrm{K} \alpha$ irradiation $(\lambda=$
$1.5406 \AA$ ) at $40 \mathrm{kV}$ and $40 \mathrm{~mA}$ in the scanning range of $2 \theta$ between $10^{\circ}$ and $90^{\circ}$. The transmission electron microscopy (TEM) used a JEM 2010 electron microscope at the accelerating voltage of $200 \mathrm{kV}$, a line resolution of $0.14 \mathrm{~nm}$, and a point resolution of $0.23 \mathrm{~nm}$. X-ray photoelectron spectroscopy (XPS) measurements were recorded in an Axis Ultra spectrometer with a monochromatized Al-K $\alpha$ X-ray source $(225 \mathrm{~W})$. The temperature programmed decomposition (TPD) was analyzed using an Auto Chem 2720 instrument with a TCD detector. The samples were pretreated in a hydrogen chloride atmosphere at a reactive temperature of $180^{\circ} \mathrm{C}$ for $4 \mathrm{~h}$. High-purity $\mathrm{N}_{2}\left(40 \mathrm{~mL} \mathrm{~min}{ }^{-1}\right)$ was then passed through the sample at $100{ }^{\circ} \mathrm{C}$ for $20 \mathrm{~min}$. The sample was heated at $10{ }^{\circ} \mathrm{C} \min ^{-1}$ from $50{ }^{\circ} \mathrm{C}$ to $800{ }^{\circ} \mathrm{C}$ during data collection. Thermogravimetric analysis (TGA) used a Netzsch STA-449 F3 Jupiter (Germany) analyzer with oxygen

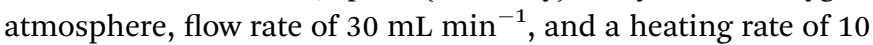
$\mathrm{K} \min ^{-1}$.

\subsection{Catalyst evaluation}

Catalytic performance during acetylene hydrochlorination was tested using a fixed-bed reactor. Nitrogen $\left(50 \mathrm{~mL} \mathrm{~min}^{-1}\right)$ was used to remove air and moisture from the reactor system before the start of the reaction. Hydrogen chloride gas at a flow rate of $20 \mathrm{~mL} \mathrm{m^{-1 }}$ was passed through the reactor to activate the catalyst $(2 \mathrm{~mL})$. The reactor was heated to $180{ }^{\circ} \mathrm{C}$, and the hydrogen chloride (33.35 $\left.\mathrm{mL} \mathrm{min}^{-1}\right)$ and acetylene (29 $\mathrm{mL} \mathrm{min}^{-1}$ ) were passed at a gas hourly space velocity (GHSV) of $870 \mathrm{~h}^{-1}$. The reaction products were detected with a GC-2014C gas chromatograph (Shimadzu).

\section{Results and discussion}

BET was used to analyze the structure of the HCS. Fig. 1a shows that the nitrogen adsorption isotherms of the HCS were consistent with the type IV curve, which indicated that the pore structures of HCS were mainly mesoporous. ${ }^{16}$ The surface area of HCS was calculated to be $762 \mathrm{~m}^{2} \mathrm{~g}^{-1}$, which is lower than that

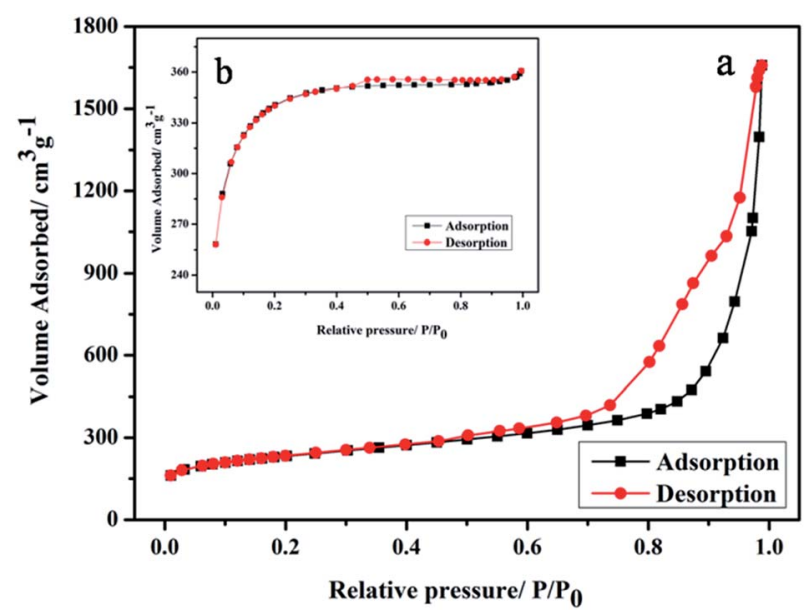

Fig. 1 Nitrogen adsorption-desorption isotherms of (a) HCS and (b) AC. 
of $\mathrm{AC}\left(1033 \mathrm{~m}^{2} \mathrm{~g}^{-1}\right)$ used as the support of Au nanoparticles in the following experiment for comparison.

XRD characterization results of $\mathrm{Au} / \mathrm{HCS}$ and $\mathrm{Au} / \mathrm{AC}$ are shown in Fig. 2. Characteristic diffraction peaks appeared at $24.4^{\circ}$, which corresponded to the (002) diffraction of the carbon material in these two catalysts. In addition, the obvious diffraction peaks at $38.5^{\circ}, 44.14^{\circ}, 64.8^{\circ}$, and $78^{\circ}$ corresponded to the (111), (200), (220), and (311) lattice planes of metallic $\mathrm{Au}^{0} .^{17}$ This result shows that the $\mathrm{Au}$ nanoparticles were successfully deposited on the HCS and AC support. The Au (111) peak was used to calculate the particle size according to the Scherrer's equation. The Au nanoparticles size in $\mathrm{Au} / \mathrm{AC}$ and $\mathrm{Au} /$ HCS catalysts were $13.7 \mathrm{~nm}$ and $6.3 \mathrm{~nm}$, respectively. The results showed that the Au nanoparticles displayed a small particle size in the Au/HCS catalyst versus the Au/AC catalyst.

The TEM images shown in Fig. 3 represent the structures of the HCS, the Au/HCS catalyst, and the Au/AC catalyst. Fig. 3a and $b$ shows that the contrast difference between the edge and the center of the spheres is obvious, indicating that the HCS was, indeed, hollow. The wall thickness was around $25 \mathrm{~nm}$, and the diameter of the core is 190-220 nm. The TEM images of Au/ HCS and Au/AC catalysts are shown in Fig. 3c-f. Fig. 3c and $\mathrm{d}$ shows that the $\mathrm{Au}$ nanoparticle dispersion is dispersed uniformly on the surface of the HCS, and some aggregation can be observed on AC. The average particle diameter of Au/HCS was $3.94 \mathrm{~nm}$ (Fig. 3h), and the average particle diameter of $\mathrm{Au} / \mathrm{AC}$ was $11.43 \mathrm{~nm}$ (Fig. 3g). This result demonstrated that the HCS support was beneficial for controlling the size of $\mathrm{Au}$ nanoparticles and is consistent with the results. ${ }^{18}$

XPS was performed because the catalytic activity of Au catalyst could be influenced by the gold valence (Fig. 4). The binding energy of $\mathrm{Au} 4 \mathrm{f}$ showed no change in the Au/AC and Au/HCS catalysts. The peaks at $84.1 \mathrm{eV}$ and $87.8 \mathrm{eV}$ were ascribed to the $4 f_{7 / 2}$ and $4 f_{5 / 2}$ peaks of metallic $A u$, respectively. ${ }^{17,19}$ No oxidative valences of $\mathrm{Au}(\mathrm{III})$ and $\mathrm{Au}(\mathrm{I})$ were found in the XPS spectras of $\mathrm{Au} / \mathrm{AC}$ and $\mathrm{Au} / \mathrm{HCS}$ catalysts, indicating all oxidative $\mathrm{Au}$ was reduced by $\mathrm{NaBH}_{4}$ reduing agent in the catalyst preparing process. The results mean that the structure of the

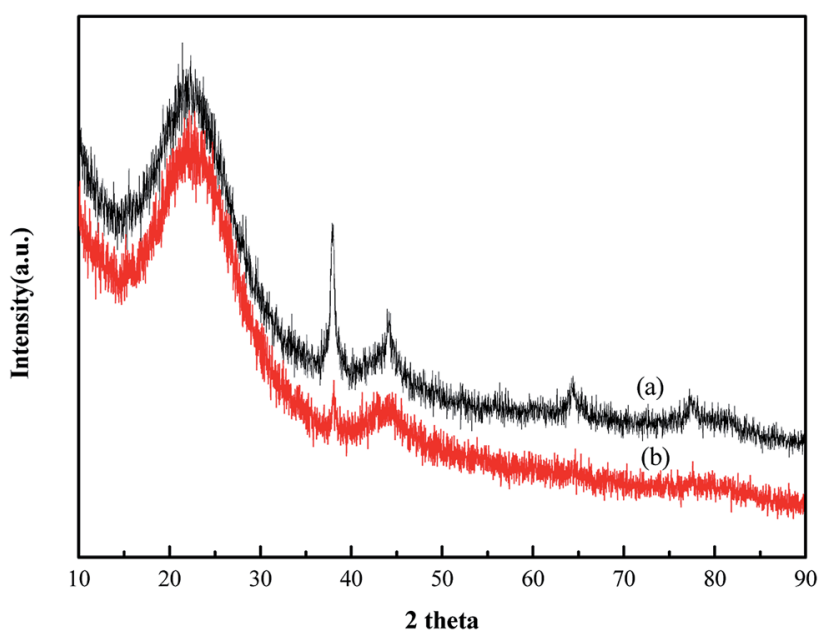

Fig. 2 XRD patterns of the (a) $1 \% \mathrm{Au} / \mathrm{AC}$ and (b) $1 \%$ Au/HCS catalysts. support had no influence on the reducing process of the $\mathrm{Au}$ precursor.

The catalytic activity of the Au/HCS and Au/AC catalysts were evaluated using a fixed-bed reactor containing $2 \mathrm{~mL}$ of the catalyst. The catalysts were evaluated under the following conditions: GHSV $=870 \mathrm{~h}^{-1}, \mathrm{~V}_{\mathrm{HCl}} / \mathrm{V}_{\mathrm{C}_{2} \mathrm{H}_{2}}=1.15$, reaction temperature $=180{ }^{\circ} \mathrm{C}$. The conversion of acetylene and selectivity of VCM during $10 \mathrm{~h}$ in the stream are shown in Fig. 5. Fig. 5b shows that the differences between HCS and AC supports have a very slight effect on the selectivity of VCM during acetylene hydrochlorination. The acetylene conversion of $\mathrm{Au} / \mathrm{AC}$ catalyst was about $57 \%$, being similar to our previous results of metallic $\mathrm{Au}$ nano-particles. ${ }^{7}$ However, acetylene conversion via the Au/HCS catalyst was much higher versus the $\mathrm{Au} / \mathrm{AC}$ catalyst (Fig. 5a); the enhanced catalytic activity of $\mathrm{Au}$ / HCS may be assigned with the good dispersion of the Au nanoparticles.

Next, TPD experiments were performed to analyze the adsorption strength of hydrogen chloride on the different supports. This study can help elucidate the mechanism underlying enhanced acetylene conversion via Au/HCS. Fig. 6 shows that the $\mathrm{Au} / \mathrm{AC}$ catalyst has two desorption peaks at $195^{\circ} \mathrm{C}$ and $410^{\circ} \mathrm{C}$. The desorption peaks in the $\mathrm{Au} / \mathrm{HCS}$ catalyst were observed at $195^{\circ} \mathrm{C}$ and $400{ }^{\circ} \mathrm{C}$. It is obvious that the second peak of the Au/HCS catalyst was larger than that of the Au/AC catalyst. The adsorption of hydrogen chloride was the ratecontrolling step in acetylene hydrochlorination. ${ }^{20}$ This phenomenon demonstrates that hydrogen chloride has stronger adsorption on the HCS during acetylene hydrochlorination than on active carbon. This might be the reason for the enhanced catalytic activity of Au/HCS.

The most important parameter of the Au catalyst for acetylene hydrochlorination was its stability under harsh reaction conditions. Therefore, we compared the running life-times of $\mathrm{Au} / \mathrm{HCS}$ and $\mathrm{Au} / \mathrm{AC}$ at $\mathrm{GHSV}=360 \mathrm{~h}^{-1}, \mathrm{~V}_{\mathrm{HCl}} / \mathrm{V}_{\mathrm{C}_{2} \mathrm{H}_{2}}=1.15$, and reaction temperature $=180{ }^{\circ} \mathrm{C}$. As shown in Fig. 7, the acetylene conversion of the $\mathrm{Au} / \mathrm{AC}$ catalyst reduced from $82 \%$ to $43 \%$ after $400 \mathrm{~h}$ of running time. The Au/HCS catalyst exhibited excellent catalytic activity for acetylene hydrochlorination, and its acetylene conversion remained above $94 \%$ even after $500 \mathrm{~h}$ running time. Considered the harsh reaction conditions (GHSV $=360$ $\mathrm{h}^{-1}$ ), the lifetime of $\mathrm{Au} / \mathrm{HCS}$ under industrial condition (GHSV $=36 \mathrm{~h}^{-1}$ ) could be predicted to be higher than previous results reported in the literatures..$^{\mathbf{2 1 , 2 2}}$

ICP-AES was used to test the actual Au loading. The actual Au loading was $0.89 \mathrm{wt} \%$ and $0.88 \mathrm{wt} \%$ in the fresh and spent $\mathrm{Au} /$ HCS catalyst, respectively. The results revealed that Au nanoparticles did not leached in the acetylene hydrochlorination process. Oxidative $\mathrm{Au}$ reduction, aggregation of $\mathrm{Au}$ nanoparticles, and carbon deposition from acetylene polymerization might be the three reasons for Au deactivation. ${ }^{7}$ However, an oxidative Au reaction could be excluded because the XPS characterization only showed metallic $\mathrm{Au}(0)$ in Au/HCS and Au/AC catalysts. Next, TGA was used to characterize the carbon deposition of the catalysts, and TEM of the spent catalysts showed the dispersion of Au nanoparticles on HCS and AC support after the lifetime testing. These experiments could help further 

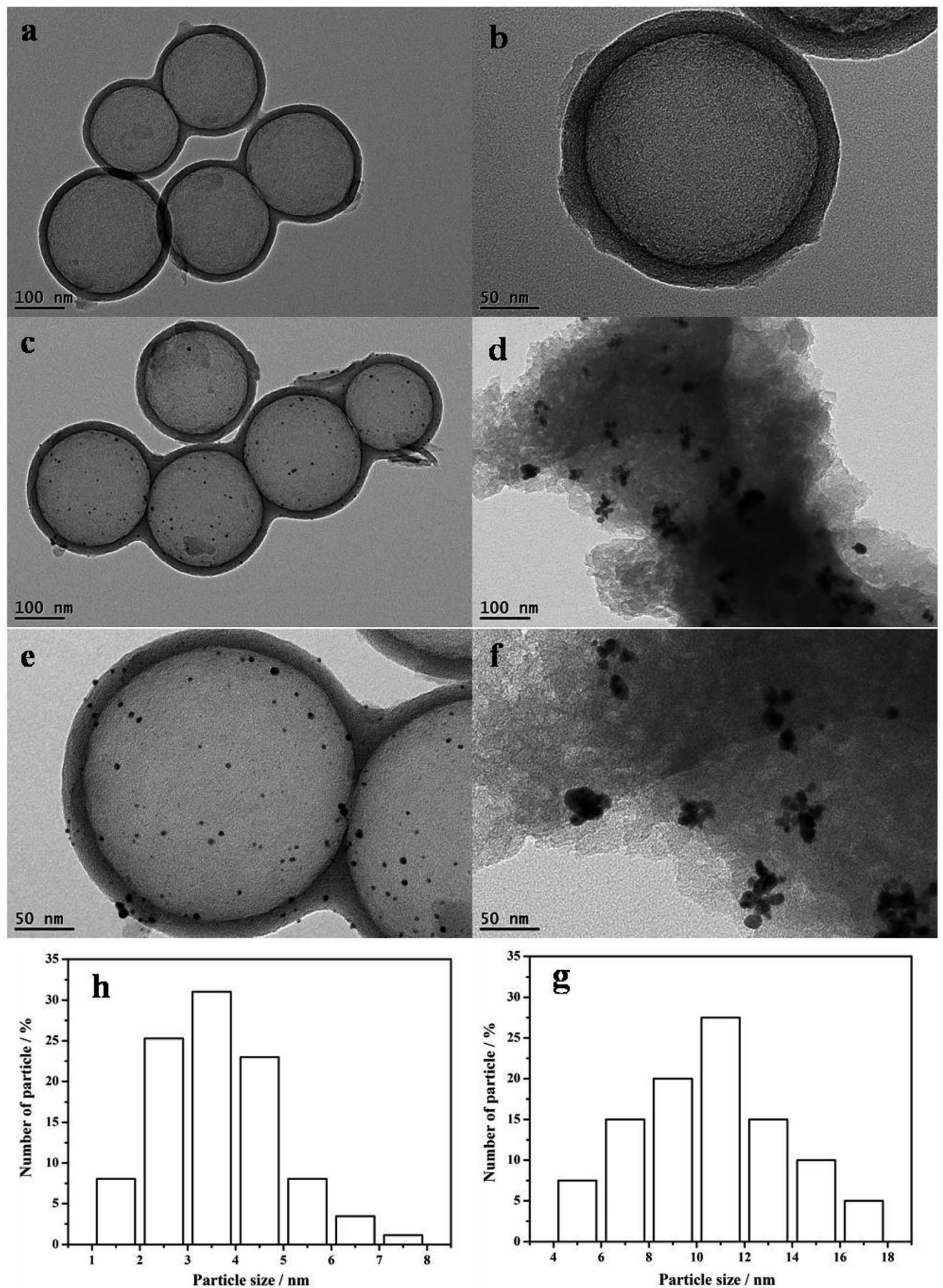

Fig. 3 TEM data on the ( $a$ and b) HCS, (c and e) Au/HCS, ( $d$ and f) Au/AC. The figure also shows the catalysts and particle size histograms of (g) Au/ $\mathrm{HCS}$ and (h) Au/C catalysts.

explain why the Au/HCS catalyst showed excellent stability for acetylene hydrochlorination. Fig. 8 indicates that the amount of carbon deposited on the Au/HCS catalyst is $1.73 \%$, and that on the $\mathrm{Au} / \mathrm{AC}$ is $4.71 \%$. Carbon deposition resulted in clogged pores and reduced surface areas in the carrier-both of these decreased catalyst activity. The carbon cokes in the Au/HCS and 


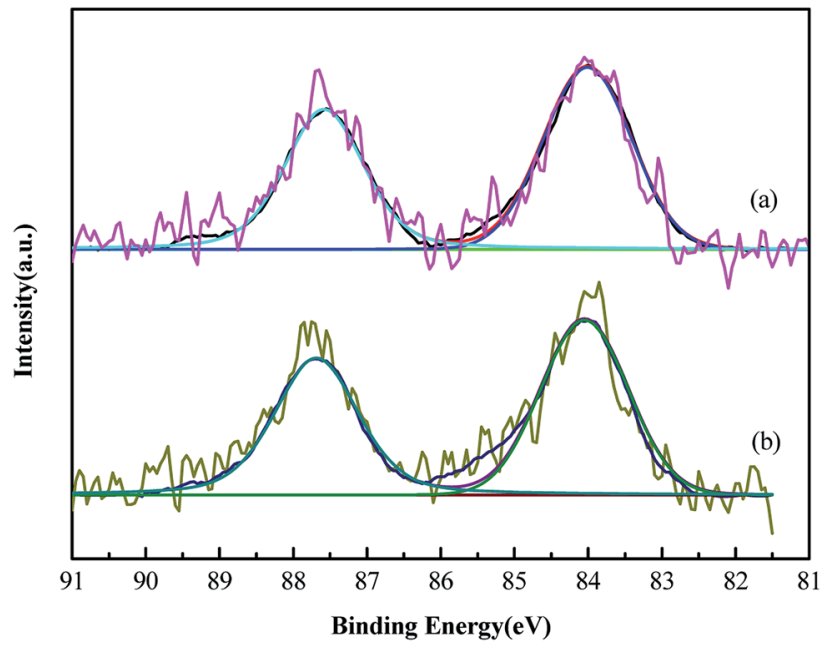

Fig. 4 XPS spectra for the Au $4 f$ of the (a) Au/AC and (b) Au/HCS catalysts.

$\mathrm{Au} / \mathrm{AC}$ were minor, and thus carbon deposition was not the main reason for the quick deactivation of $\mathrm{Au} / \mathrm{AC}$ catalyst. TEM was performed to determine whether the $\mathrm{Au}$ particle aggregation was the main reason for the deactivation rapid of the $\mathrm{Au} / \mathrm{AC}$ catalyst (Fig. 9). Fig. 9b shows the spent $\mathrm{Au} / \mathrm{AC}$ catalyst-the particle size of Au nanoparticles obviously increased after $400 \mathrm{~h}$ running, which indicates that the $\mathrm{Au}$ nanoparticles form aggregates during acetylene hydrochlorination. This indicates that aggregation of the $\mathrm{Au}$ nanoparticles was the main reason for the deactivation of the $\mathrm{Au} / \mathrm{AC}$ catalyst. However, no obvious aggregation was seen in Au/HCS. The Au nanoparticles were still uniformly dispersed even after $500 \mathrm{~h}$ running, which means that the hollow structure of HCS could restrict the aggregation of $\mathrm{Au}$ nanoparticles during the acetylene hydrochlorination process. Mesopores originated from the decomposition of micelles could protect the interior $\mathrm{Au}$ nano-particles from aggregating with $\mathrm{Au}$ in other hollow carbon. ${ }^{23}$ In addition, the hollow shell of HCS can act as a barrier, which may be another

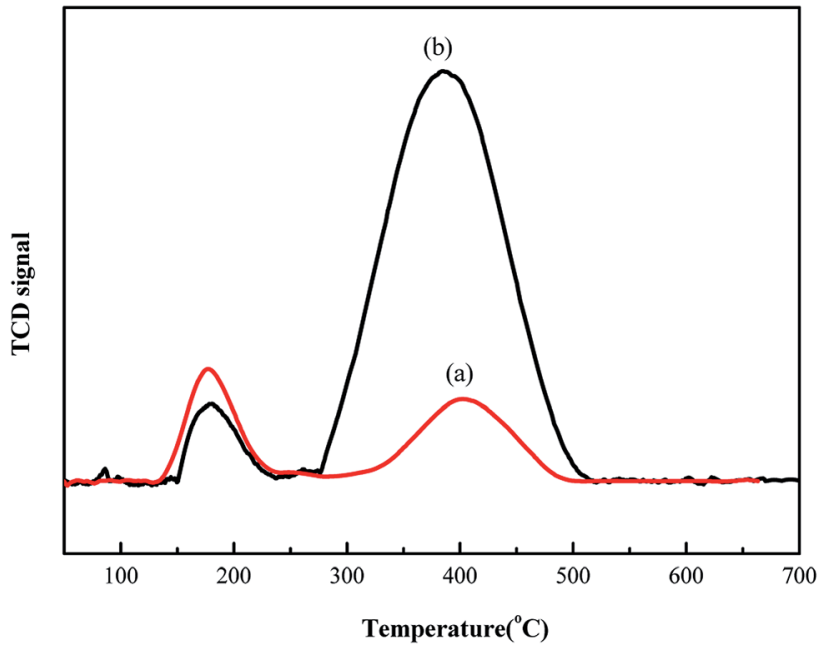

Fig. 6 TPD-HCl profiles of the (a) Au/AC and (b) Au/HCS catalysts.

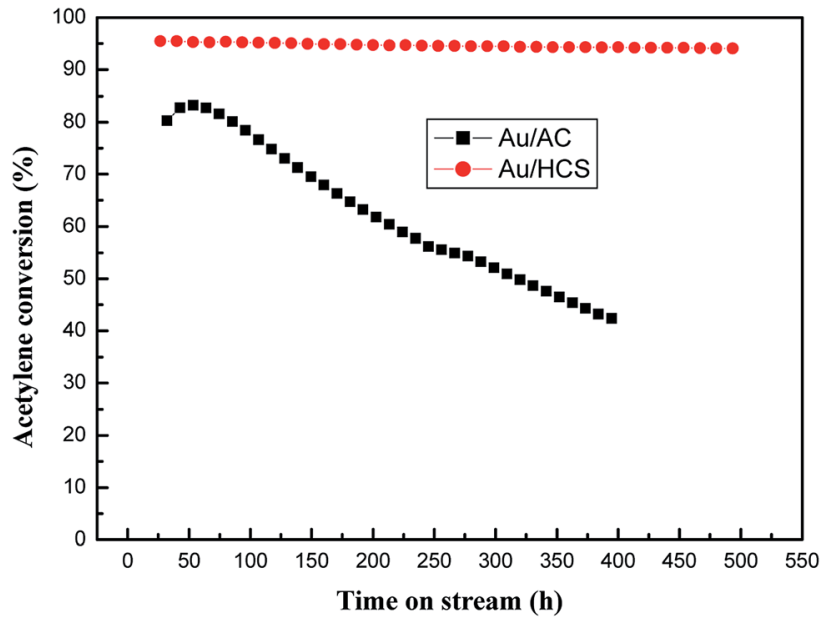

Fig. 7 Long lifetime testing of $\mathrm{Au} / \mathrm{AC}$ and $\mathrm{Au} / \mathrm{HCS}$ catalysts (GHSV = $360 \mathrm{~h}^{-1}, \mathrm{~V}_{\mathrm{HCl}} / \mathrm{V}_{\mathrm{C}_{2} \mathrm{H}_{2}}=1.15$, reaction temperature $\left.=180^{\circ} \mathrm{C}\right)$.
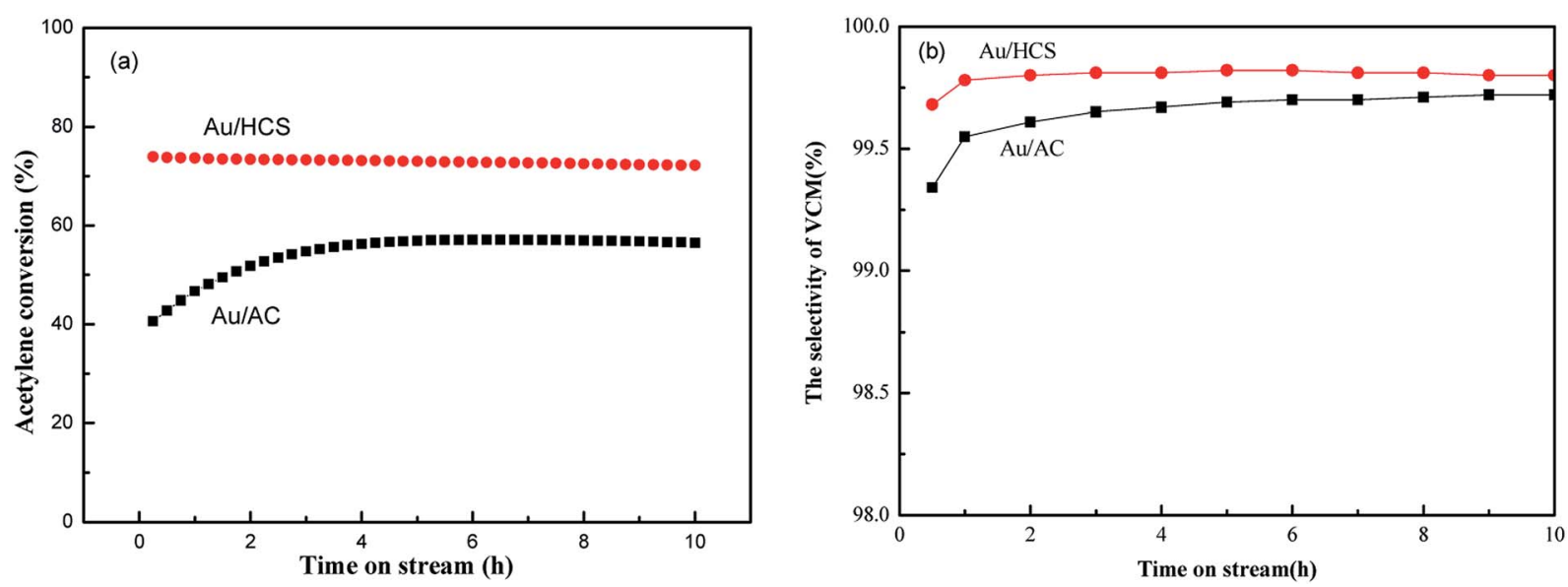

Fig. 5 Conversion of acetylene (a) and selectivity of VCM (b) in acetylene hydrochlorination catalyzed by the Au/HCS and Au/AC catalysts (GHSV $=870 \mathrm{~h}^{-1}, \mathrm{~V}_{\mathrm{HCl}} / \mathrm{V}_{\mathrm{C}_{2} \mathrm{H}_{2}}=1.15$, reaction temperature $=180^{\circ} \mathrm{C}$ ). 

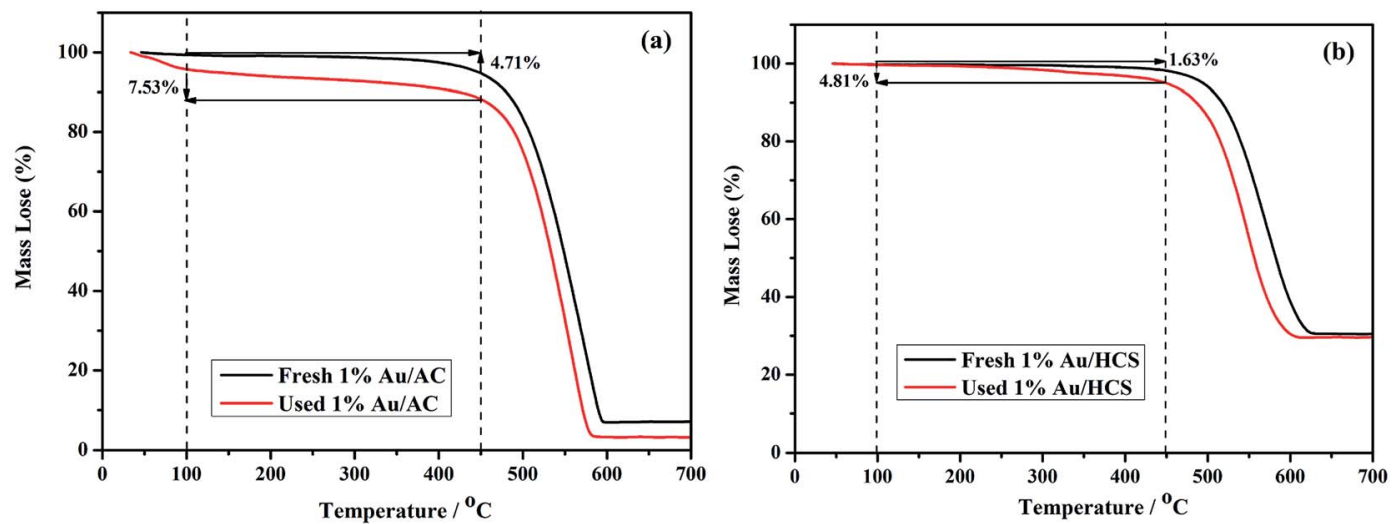

Fig. 8 TGA curves of (a) Au/AC and (b) Au/HCS catalysts.
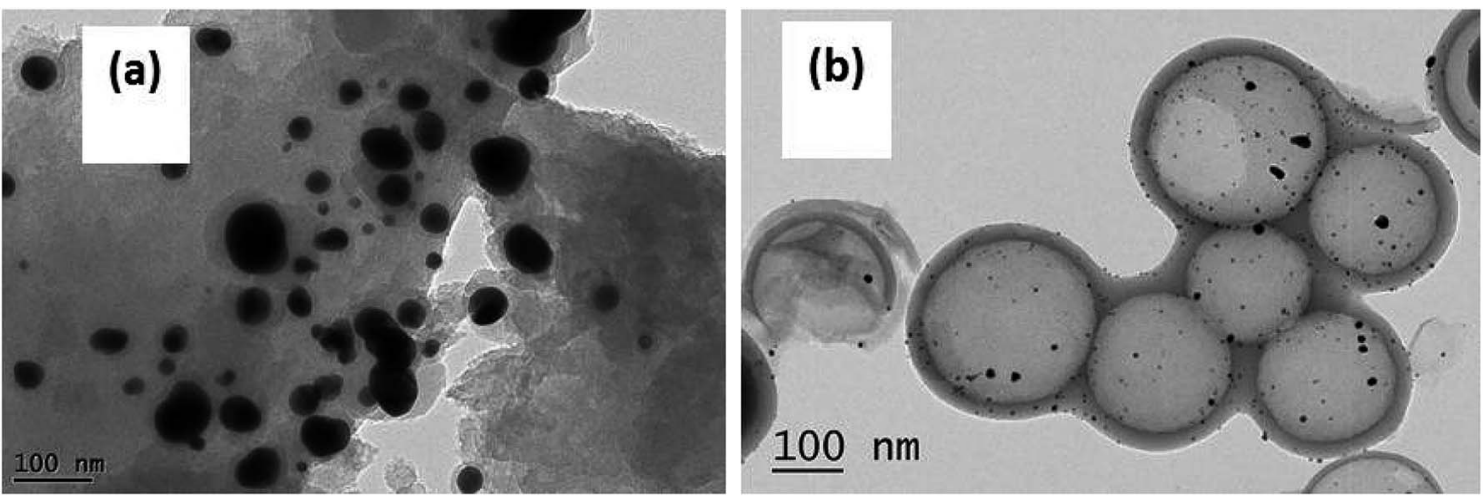

Fig. 9 TEM images of the (a) spent Au/AC and (b) Au/HCS catalysts.

reason for the weill dispersion of Au nanoparticles. ${ }^{24}$ The good dispersion of the Au nanoparticles due to the HCS structure may be the reason for the excellent stability of the Au/HCS catalyst.

\section{Conclusions}

The Au/HCS catalyst was successfully prepared and applied to the acetylene hydrochlorination reaction. Versus an AC support, the HCS support controlled the Au nanoparticle size to a mean of $3.94 \mathrm{~nm}$, improved the dispersion of the Au particles, and inhibited agglomeration. Au/HCS displayed excellent catalytic activity and stability for acetylene hydrochlorination. The acetylene conversion of Au/HCS can be maintained above $92 \%$ even after $500 \mathrm{~h}$ lifetime running. The excellent catalytic performance of $\mathrm{Au} / \mathrm{HCS}$ was attributed to the presence of the HCS support that limits the aggregation of $\mathrm{Au}$ nanoparticles.

\section{Conflicts of interest}

There are no conflicts to declare.

\section{Acknowledgements}

This work was supported by National Natural Science Funds of China (NSFC, U1703352), the State Key Research and
Development Project of China (2016YFB0301603), and the International Corporation of S\&T Project in Xinjiang Bingtuan (2018BC003) and the International Corporation of S\&T Project in Shihezi University (GJHZ201701).

\section{References}

1 J. W. Zhong, Y. P. Xu and Z. M. Liu, Heterogeneous non-mercury catalysts for acetylene hydrochlorination: progress, challenges, and opportunities, Green Chem., 2018, 20, 2412-2427.

2 G. J. Hutchings, Vapor phase hydrochlorination of acetylene: correlation of catalytic activity of supported metal chloride catalysts, J. Catal., 1985, 98, 292-295.

3 P. Johnston, N. Carthey and G. J. Hutchings, Discovery, Development, and Commercialization of Gold Catalysts for Acetylene Hydrochlorination, J. Am. Chem. Soc., 2015, 137, 14548-14557.

4 G. Malta, S. A. Kondrat, S. J. Freakley, C. J. Davies, L. Lu, S. Dawson, A. Thetford, E. K. Gibson, D. J. Morgan, W. Jones, P. P. Wells, P. Johnston, C. R. A. Catlow, C. J. Kiely and G. J. Hutchings, Identification of single-site gold catalysis in acetylene hydrochlorination, Science, 2017, 355, 1399-1402.

5 G. Malta, S. A. Kondrat, S. J. Freakley, C. J. Davies, S. Dawson, X. Liu, L. Lu, K. Dymkowski, F. Fernandez-Alonso, 
S. Mukhopadhyay, E. K. Gibson, P. P. Wells, S. F. Parker, C. J. Kiely and G. J. Hutchings, Deactivation of a Single-Site Gold-on-Carbon Acetylene Hydrochlorination Catalyst: An X-ray Absorption and Inelastic Neutron Scattering Study, ACS Catal., 2018, 8, 8493-8505.

6 S. K. Kaiser, R. H. Lin, S. Mitchell, E. Fako, F. Krumeich, R. Hauert, O. V. Safonova, V. A. Kondratenko, E. V. Kondratenko, S. M. Collins, P. A. Midgley, N. Lopez and J. Perez-Ramirez, Controlling the speciation and reactivity of carbon-supported gold nanostructures for catalysed acetylene hydrochlorination, Chem. Sci., 2019, 10, 359-369.

7 B. Dai, Q. Q. Wang, F. Yu and M. Y. Zhu, Effect of Au nanoparticle aggregation on the deactivation of the $\mathrm{AuCl}_{3} / \mathrm{AC}$ catalyst for acetylene hydrochlorination, Sci. Rep., 2015, 5, 10553.

8 B. Dai, X. Y. Li, J. L. Zhang, F. Yu and M. Y. Zhu, Application of mesoporous carbon nitride as a support for an Au catalyst for acetylene hydrochlorination, Chem. Eng. Sci., 2015, 135, 472-478.

9 J. Liu, G. J. Lan, Y. Y. Qiu, X. L. Wang and Y. Li, Wheat flourderived N-doped mesoporous carbon extrudes as an efficient support for Au catalyst in acetylene hydrochlorination, Chin. J. Catal., 2018, 39, 1664-1671.

10 L. Ye, X. P. Duan, S. Wu, T. S. Wu, Y. X. Zhao, A. W. Robertson, H. L. Chou, J. W. Zheng, T. Ayvali, S. Day, C. Tang, Y. Soo, Y. Z. Yuan and S. C. E. Tsang, Selfregeneration of $\mathrm{Au} / \mathrm{CeO}_{2}$ based catalysts with enhanced activity and ultra-stability for acetylene hydrochlorination, Nat. Commun., 2019, 10, 914.

11 K. Chen, L. H. Kang, M. Y. Zhu and B. Dai, Mesoporous carbon with controllable pore sizes as a support of the $\mathrm{AuCl}_{3}$ catalyst for acetylene hydrochlorination, Catal. Sci. Technol., 2015, 5, 1035-1040.

12 J. H. Xu, J. Zhao, J. T. Xu, T. T. Zhang, X. N. Li, X. X. Di, J. Ni, J. G. Wang and J. Cen, Influence of Surface Chemistry of Activated Carbon on the Activity of Gold/Activated Carbon Catalyst in Acetylene Hydrochlorination, Ind. Eng. Chem. Res., 2014, 53, 14272-14281.

13 J. Wu, F. P. Hu, X. D. Hu, Z. D. Wei and P. K. Shen, Improved kinetics of methanol oxidation on $\mathrm{Pt} /$ hollow carbon sphere catalysts, Electrochim. Acta, 2008, 53, 8341-8345.

14 J. Zhang, L. Ma, M. Y. Gan, F. F. Yang, S. N. Fu and X. Li, Well-dispersed platinum nanoparticles supported on hierarchical nitrogen-doped porous hollow carbon spheres with enhanced activity and stability for methanol electrooxidation, J. Power Sources, 2015, 288, 42-52.

15 B. Y. Guan, X. Wang, Y. Xiao, Y. L. Liu and Q. S. Huo, A versatile cooperative template-directed coating method to construct uniform microporous carbon shells for multifunctional coreshell nanocomposites, Nanoscale, 2013, 5, 2469-2475.

16 I. Nongwe, V. Rivat, R. Meijboom and N. J. Coville, Pt supported nitrogen doped hollow carbon spheres for the catalysed reduction of cinnamaldehyde, Appl. Catal., A, 2016, 517, 30-38.

17 C. F. Huang, M. Y. Zhu, L. H. Kang, X. Y. Li and B. Dai, Active carbon supported $\mathrm{TiO}_{2}-\mathrm{AuCl}_{3} / \mathrm{AC}$ catalyst with excellent stability for acetylene hydrochlorination reaction, Chem. Eng. J., 2014, 242, 69-75.

18 Z. X. Yan, J. M. Xie, S. K. Zong, M. M. Zhang, Q. Sun and M. Chen, Small-sized Pt particles on mesoporous hollow carbon spheres for highly stable oxygen reduction reaction, Electrochim. Acta, 2013, 109, 256-261.

19 Y. W. Chen, H. J. Chen and D. S. Lee, $\mathrm{Au} / \mathrm{Co}_{3} \mathrm{O}_{4}-\mathrm{TiO}_{2}$ catalysts for preferential oxidation of $\mathrm{CO}$ in $\mathrm{H}-2$ stream, $J$. Mol. Catal. A: Chem., 2012, 363, 470-480.

20 M. Conte, A. F. Carley, C. Heirene, D. J. Willock, P. Johnston, A. A. Herzing, C. J. Kiely and G. J. Hutchings, Hydrochlorination of acetylene using a supported gold catalyst: a study of the reaction mechanism, J. Catal., 2007, 250, 231-239.

21 H. Y. Zhang, B. Dai, W. Li, X. G. Wang, J. L. Zhang, M. Y. Zhu and J. J. Gu, Non-mercury catalytic acetylene hydrochlorination over spherical activated-carbon-supported $\mathrm{Au}-\mathrm{Co}(\mathrm{III})-\mathrm{Cu}(\mathrm{II})$ catalysts, J. Catal., 2014, 316, 141-148.

22 Y. Z. Dong, H. Y. Zhang, W. Li, M. X. Sun, C. L. Guo and J. L. Zhang, Bimetallic Au-Sn/AC catalysts for acetylene hydrochlorination, J. Ind. Eng. Chem., 2016, 35, 177-184.

23 H. Y. Qian, J. Tang, M. S. A. Hossain, Y. Bando and X. Wang, Localization of platinum nanoparticles on inner walls of mesoporous hollow carbon spheres for improvement of electrochemical stability, Nanoscale, 2017, 9, 16264-16272.

24 R. Shi, J. Wang, J. Zhao, S. Liu, P. Hao, Z. Li and J. Ren, Cu nanoparticles encapsulated with hollow carbon spheres for methanol oxidative carbonylation: tuning of the catalytic properties by particle size control, Appl. Surf. Sci., 2018, 459, 707-715. 